\title{
Dynamic Nuclear Polarization of Long- Lived Nuclear Spin States in Methyl Groups
}

Jean-Nicolas Dumez, Basile Vuichoud, Daniele Mammoli, Aurélien Bornet, Arthur C. Pinon, Gabriele Stevanato, Benno Meier, Geoffrey Bodenhausen, Sami Jannin, and Malcolm H Levitt

J. Phys. Chem. Lett., Just Accepted Manuscript • DOI: 10.1021/acs.jpclett.7b01512 • Publication Date (Web): 14 Jul 2017

Downloaded from http://pubs.acs.org on July 17, 2017

\section{Just Accepted}

"Just Accepted" manuscripts have been peer-reviewed and accepted for publication. They are posted online prior to technical editing, formatting for publication and author proofing. The American Chemical Society provides "Just Accepted" as a free service to the research community to expedite the dissemination of scientific material as soon as possible after acceptance. "Just Accepted" manuscripts appear in full in PDF format accompanied by an HTML abstract. "Just Accepted" manuscripts have been fully peer reviewed, but should not be considered the official version of record. They are accessible to all readers and citable by the Digital Object Identifier (DOI®). "Just Accepted" is an optional service offered to authors. Therefore, the "Just Accepted" Web site may not include all articles that will be published in the journal. After a manuscript is technically edited and formatted, it will be removed from the "Just Accepted" Web site and published as an ASAP article. Note that technical editing may introduce minor changes to the manuscript text and/or graphics which could affect content, and all legal disclaimers and ethical guidelines that apply to the journal pertain. ACS cannot be held responsible for errors or consequences arising from the use of information contained in these "Just Accepted" manuscripts. 


\title{
Dynamic Nuclear Polarization of Long-Lived Nuclear Spin States in Methyl Groups
}

\author{
Jean-Nicolas Dumez, ${ }^{1}$ Basile Vuichoud, ${ }^{2,3}$ Daniele Mammoli, ${ }^{2}$ Aurélien Bornet, ${ }^{2,3}$ Arthur C. \\ Pinon, ${ }^{2}$ Gabriele Stevanato, ${ }^{2}$ Benno Meier, ${ }^{4}$ Geoffrey Bodenhausen, ${ }^{5}$ Sami Jannin, ${ }^{2,3}$ Malcolm H. \\ Levitt $^{4 *}$ \\ ${ }^{1}$ Institut de Chimie des Substances Naturelles, CNRS UPR2301, Univ. Paris Sud, Université \\ Paris-Saclay, 91190 Gif-sur-Yvette, France. \\ ${ }^{2}$ Institut des Sciences et Ingénierie Chimiques, Ecole Polytechnique Fédérale de Lausanne \\ (EPFL), CH-1015 Lausanne, Switzerland. \\ ${ }^{3}$ Université de Lyon, CNRS, Université Claude Bernard Lyon 1, ENS de Lyon, Institut des \\ Sciences Analytiques, UMR 5280, 69100 Villeurbanne, France \\ ${ }^{4}$ School of Chemistry, University of Southampton, Southampton, SO17 1BJ, UK \\ ${ }^{5}$ Laboratoire des Biomolécules, CNRS-ENS-UPMC UMR7203, 75005 Paris, France
}

\section{Corresponding Author}

Email: $\underline{\text { mhl@soton.ac.uk }}$ 
We have induced hyperpolarized long-lived states in compounds containing ${ }^{13} \mathrm{C}$-bearing methyl groups by dynamic nuclear polarization (DNP) at cryogenic temperatures, followed by dissolution with a warm solvent. The hyperpolarized methyl long-lived states give rise to enhanced antiphase ${ }^{13} \mathrm{C}$ NMR signals in solution, which often persist for times much longer than the ${ }^{13} \mathrm{C}$ and ${ }^{1} \mathrm{H}$ spin-lattice relaxation times under the same conditions. The DNP-induced effects are similar to quantum-rotor-induced-polarization (QRIP), but are observed in a wider range of compounds, since they do not depend critically on the height of the rotational barrier. We interpret our observations with a model in which nuclear-Zeeman and methyl-tunnelling reservoirs adopt an approximately uniform temperature, under DNP conditions. The generation of hyperpolarized NMR signals which persist for relatively long times in a range of methylbearing substances may be important for applications such as investigations of metabolism, enzymatic reactions, protein-ligand binding, drug screening, and molecular imaging.

\section{TOC GRAPHICS}

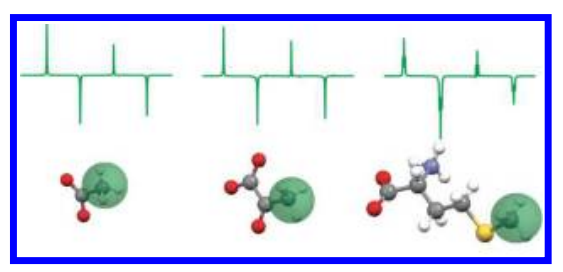


Nuclear magnetic resonance (NMR) is a highly versatile and informative analytical technique, with applications ranging from anatomic imaging to atomic-scale analysis of molecular structure and dynamics. The main limitation of NMR is arguably its low sensitivity, which originates in the small population differences between spin energy levels in thermal equilibrium, at even the highest static magnetic fields available. A range of hyperpolarization methods is available for increasing the NMR sensitivity by boosting the polarization. In solution, large enhancements are generated by Dissolution Dynamic Nuclear Polarization (d-DNP) and Parahydrogen Induced Polarization (PHIP). ${ }^{1-3}$ The main drawback of these methods is that once generated, the hyperpolarization decays on a timescale governed by the longitudinal relaxation time, $T_{1}$, which is an irreversible process.

Long-lived nuclear spin states (LLS) offer promise for alleviating this limitation. LLS are defined as nuclear spin configurations that relax much more slowly than longitudinal magnetization. ${ }^{4-6}$ LLS exist for groups of two spins or more, when the effects of fluctuating spin interactions are cancelled or reduced for symmetry reasons. The prototypical long-lived state, also known as singlet order, is the population difference between the singlet and triplet manifolds in an ensemble of spin-1/2 pairs. Potential applications of the LLS concept include drug screening, molecular imaging and reaction monitoring. ${ }^{7-9}$ LLS have been used as a carrier of hyperpolarisation. ${ }^{10-15}$ Molecular scaffolds have been designed to minimise the relaxation rates of singlet order, with reported lifetimes of over an hour. ${ }^{16}$ Such record lifetimes, however, concern chemical motifs that are not commonly found.

Long-lived nuclear spin states are also known to exist in methyl, $\mathrm{CH}_{3}$ groups, ${ }^{17-18}$ which are ubiquitous in organic chemistry and biochemistry. Methyl LLS correspond to a population imbalance between manifolds of states of different spin permutation symmetry, conventionally 
denoted as $\mathrm{A}$ and $\mathrm{E}$ manifolds; we refer to the relevant population difference as an $\mathrm{A} / \mathrm{E}$ imbalance (AEI). Such a population imbalance is protected from dominant relaxation mechanisms by rapid methyl rotation, leading to long lifetimes in solution.

Methyl LLS are implicated in the phenomenon of quantum-rotor-induced polarization (QRIP), in which strong antiphase ${ }^{13} \mathrm{C}$ signals are observed when certain compounds are cooled to cryogenic temperatures, rapidly dissolved in a hot solvent, and observed by solution NMR at room temperature. ${ }^{19-20}$ QRIP has been observed for compounds such as $\gamma$-picoline (4methylpyridine) for which the methyl rotation encounters an unusually low rotational barrier, leading to a significant tunnelling splitting of $\sim 6 \mathrm{~K}$ between the $\mathrm{A}$ and $\mathrm{E}$ manifolds in the cryogenic solid state. For such special cases, a significant AEI may be established simply by equilibrating the sample at a temperature below $10 \mathrm{~K}$, without any paramagnetic agents or microwaves. This population imbalance builds up within tens of minutes to a value that is significantly larger than the Zeeman polarization at room temperature. The AEI is maintained to a significant degree through the dissolution process, leading to the generation of a hyperpolarized methyl LLS in the room temperature solution, and hence enhanced antiphase ${ }^{13} \mathrm{C}$ signals through cross-relaxation. $^{17}$

In this paper, we show that the requirement of low rotational barriers (or, equivalently, large tunnelling splittings) required for QRIP at liquid-helium temperature may be alleviated by using dynamic nuclear polarization (DNP). This opens up the practical use of methyl AEI and in turn long-lived states to arbitrary methyl-bearing molecules. We report observations of this effect for compounds containing methyl groups in several molecular environments. The interplay between quantum-rotor and DNP effects is illustrated by simple energy-level diagrams. 

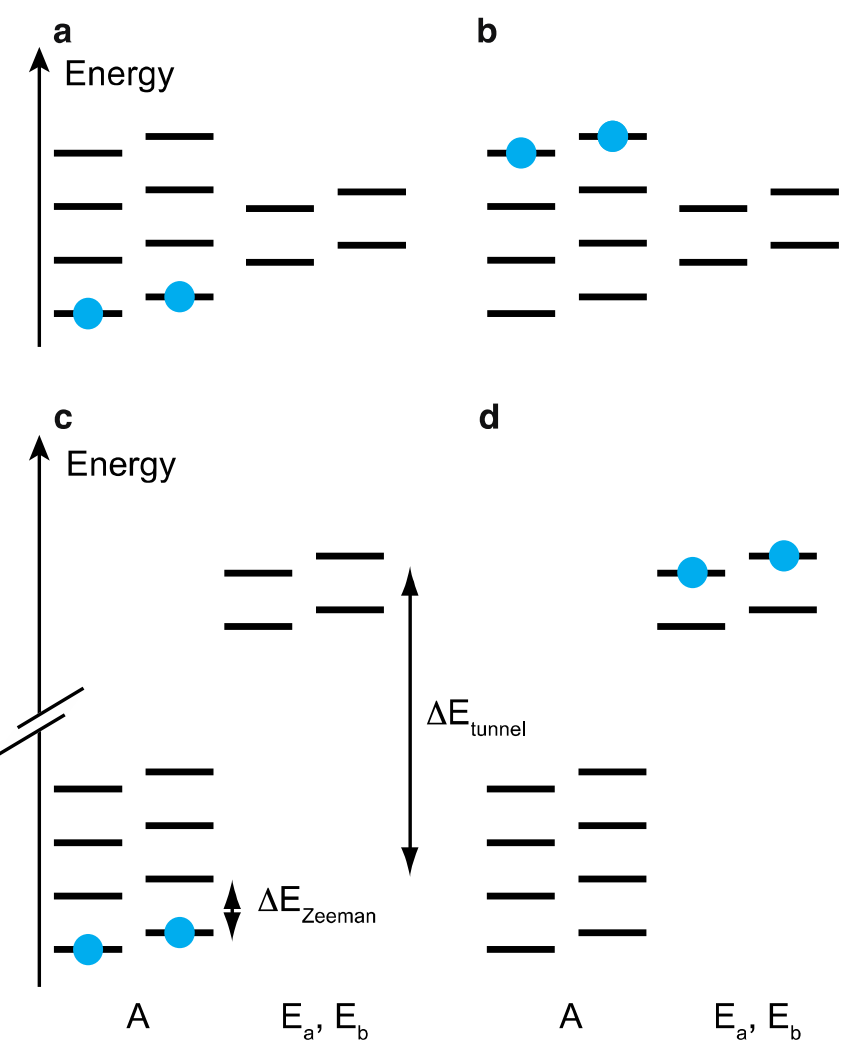

Fig. 1 Schematic energy-level diagram for a methyl group, at liquid-helium temperature, with a vanishing (a-b) or large (c-d) tunnelling splitting (only the lowest tunnelling levels are shown). Exaggerated spin populations are shown for positive DNP in a and $\mathbf{c}$, and negative DNP in $\mathbf{b}$ and d.

Consider a ${ }^{13} \mathrm{C}$-bearing methyl group in a solid. The $\mathrm{A}_{3} \mathrm{X}$ spin system consists of three protons and one carbon-13, with $2^{4}=16$ energy levels. Provided that the three protons are magnetically equivalent, i.e., the rapid methyl rotation averages the chemical shift anisotropy of the three protons and their dipolar couplings with external spins, the energy eigenstates are given by symmetry-adapted combinations of these energy levels, which transform as irreducible representations of the group $\mathrm{C} 3(\mathrm{M})$, with 8 combinations of symmetry A, and 8 of symmetry $\mathrm{E}$ (see ref. ${ }^{17}$ for a detailed description). In general, the $\mathrm{A}$ and $\mathrm{E}$ levels are split by the ${ }^{1} \mathrm{H}$ and ${ }^{13} \mathrm{C}$ Zeeman splittings, while the E levels are raised in energy with respect to the A levels by the 
tunnelling splitting. This splitting is associated with the overlap of the spatial wave-functions of the methyl protons, and depends strongly on the methyl rotational barrier. ${ }^{21}$ Two extreme cases may be identified: in the case of a large rotational barrier, the tunnelling splitting is very small, and the energy level structure is dominated by the Zeeman splittings (Fig. 1a-b). In the case of a small rotational barrier, on the other hand, the tunnelling splitting may be much larger than the Zeeman splitting (Fig. 1c-d). In extreme cases such as 4-methylpyridine ( $\gamma$-picoline), the tunnelling splitting is $\sim 126 \mathrm{GHz}$, which is more than 2 orders of magnitude larger than the ${ }^{1} \mathrm{H}$ nuclear Zeeman splitting in accessible magnetic fields.

In the case of a very large tunnelling splitting (Fig. 1c-d), a large and positive AEI may be established by allowing the sample to reach thermal equilibrium at a temperature of a few Kelvin by cooling with liquid He. This resulting positive AEI is substantially maintained through the dissolution process, leading to a hyperpolarized methyl LLS that gives rise to enhanced hyperpolarized ${ }^{13} \mathrm{C}$ signals through cross-relaxation. This is the origin of QRIP. ${ }^{17-20}$ However, in the case of a high rotational barrier and a small tunnelling splitting (Fig. 1a-b), cooling by liquid He leads to a small or absent AEI. Conventional QRIP experiments fail for such systems unless the sample temperature is lowered well below $1 \mathrm{~K}$, where equilibration of the sample may take prohibitively long times.

DNP may be used to establish Zeeman population imbalances of arbitrary signs and far from thermal equilibrium values. ${ }^{22}$ This has been used to generate hyperpolarized long-lived states in pairs of spins with $I=1 / 2 \cdot{ }^{8,23-24}$ Similarly, in the case of methyl groups, we show that DNP can give rise to significant AEI, by preferentially populating one manifold with respect to the other, even when these groups are not shifted with respect to each other by a large tunnelling splitting. As in the QRIP effect, the AEI in the solid state translates into a hyperpolarized LLS in solution, 
which in turn gives rise to hyperpolarized antiphase ${ }^{13} \mathrm{C}$ signals in solution. Related effects have been observed for materials containing $\mathrm{CD}_{3}$ groups. $^{25}$

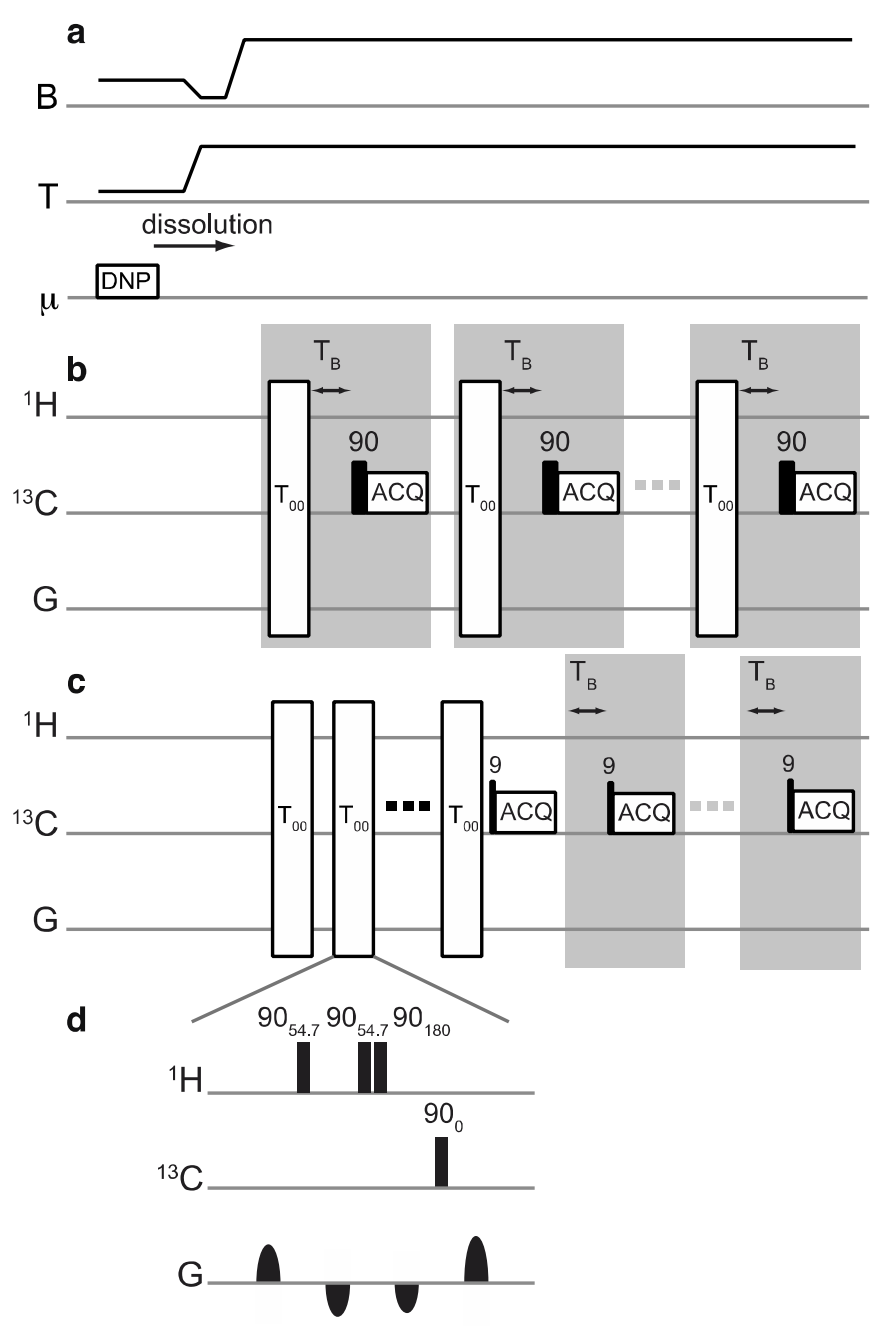

Fig. 2 Experimental procedure for the dissolution-NMR experiments. After polarization in the solid-state, the sample is dissolved and transferred to the high-resolution liquid-state NMR system; the evolution of the magnetic field and the temperature is shown in a. For the acquisition, in $\mathbf{b}$, the following sequence of event is looped: $T_{00}$ filter, build-up delay $T_{B}, 90^{\circ}$ pulse, acquisition. In c, the $T_{00}$ filter is looped $N_{\text {filter }}$ times, and a series of spectra is acquired with 
small-tip-angle pulses. The $T_{00}$ filter is composed of the sequence of pulses and gradients shown in $\mathbf{d}$. Repeated acquisition events are shown within light grey boxes.

The experimental procedure used to demonstrate this effect is sketched in Fig. 2. The species of interest are prepared in a glass-forming solvent $\left(100 \mu \mathrm{L}\right.$ of $\mathrm{D}_{2} \mathrm{O}$ :glycerol- $d_{8} 1: 1$ with $50 \mathrm{mM}$ TEMPOL), cooled to a temperature of $\sim 1.2 \mathrm{~K}$ in a field of $6.7 \mathrm{~T}$ and irradiated with frequencymodulated microwaves, ${ }^{26-28}$ with a frequency slightly displaced from the centre of the electron spin resonance line. The polarization and the AEI were allowed to build up for 20 minutes. The Zeeman polarization was found to be $P\left({ }^{1} \mathrm{H}\right) \sim 50 \%$ for positive DNP (microwave frequency at $f_{\mu \text { waves }}=187.8 \mathrm{GHz}$, below the centre of the electron spin resonance line) and $P\left({ }^{1} \mathrm{H}\right) \sim-50 \%$ for negative DNP (microwave frequency at $f_{\mu \text { waves }}=188.3 \mathrm{GHz}$, above the centre of the electron spin resonance line).

Once the polarization and AEI are established, the dissolution-NMR experiment is carried out. The DNP sample is i) dissolved with $\mathrm{D}_{2} \mathrm{O}(5 \mathrm{~mL}$ heated to $c a .420 \mathrm{~K}$ at a pressure of $1 \mathrm{MPa})$ in $700 \mathrm{~ms}$, then ii) pushed in $4.5 \mathrm{~s}$ with a pressure of $0.6 \mathrm{MPa}$ He gas to a home-built injector in a 11.7 $\mathrm{T}$ magnet, and iii) finally injected in ca. $2 \mathrm{~s}$ in a $5-\mathrm{mm}$ sample tube (complete sequence 7.2 s). The TEMPOL concentration in the final sample is $1 \mathrm{mM}$. The AEI is NMR silent, but "bleeds" by cross-relaxation into spin-state population differences that give rise to an antiphase ${ }^{13} \mathrm{C}$ multiplet upon application of a radiofrequency pulse on the ${ }^{13} \mathrm{C}_{\text {channel }}{ }^{17-18}$. Fig. $2 \mathrm{~b}$ shows the pulse sequence used to identify methyl LLS and measure relaxation rates. After injection of the sample in the high-resolution NMR system, a series of ${ }^{13} \mathrm{C}$ spectra is obtained, with a repetition time of $5 \mathrm{~s}$. Before each acquisition, a combination of RF pulses and gradients known as " $T_{00}$ filter" is used to suppress signals arising from any components of the spin density matrix other than the AEI (the notation " $T_{00}$ " filter reflects the suppression of density operator terms that 
do not transform as spherical tensor operators of rank 0 and component index 0$)^{29}$. The spin system is then left to evolve for a relaxation delay, during which the AEI converts partially to population differences across observable transitions. A strong, non-selective $90^{\circ}$ excitation pulse is applied on the ${ }^{13} \mathrm{C}$ channel before acquisition.

In the case of $2-{ }^{13} \mathrm{C}$-acetate, $3-{ }^{13} \mathrm{C}$-pyruvate and ${ }^{13} \mathrm{CH}_{3}$-methionine, this procedure leads to an enhanced antiphase ${ }^{13} \mathrm{C}$ multiplet, as shown in Fig. 3a-c, indicating that a significant AEI is generated by dynamic nuclear polarization; no such signal is observed for $3-{ }^{13} \mathrm{C}$-alanine (not shown). In the case of $2-{ }^{13} \mathrm{C}$-acetate, small antiphase signals are also observed when no microwave irradiation is applied under cryogenic conditions (Fig.3g); no such signals are observed for the three other compounds (Fig. 3h-i). Changing the microwave irradiation frequency to the opposite side of the electron spin resonance line inverts the sign of the antiphase ${ }^{13} \mathrm{C}$ signals for $2-{ }^{13} \mathrm{C}$-acetate, but not for ${ }^{13} \mathrm{CH}_{3}$-methionine and $3-{ }^{13} \mathrm{C}$-pyruvate (Fig. $3 \mathrm{~d}-\mathrm{f}$ ).
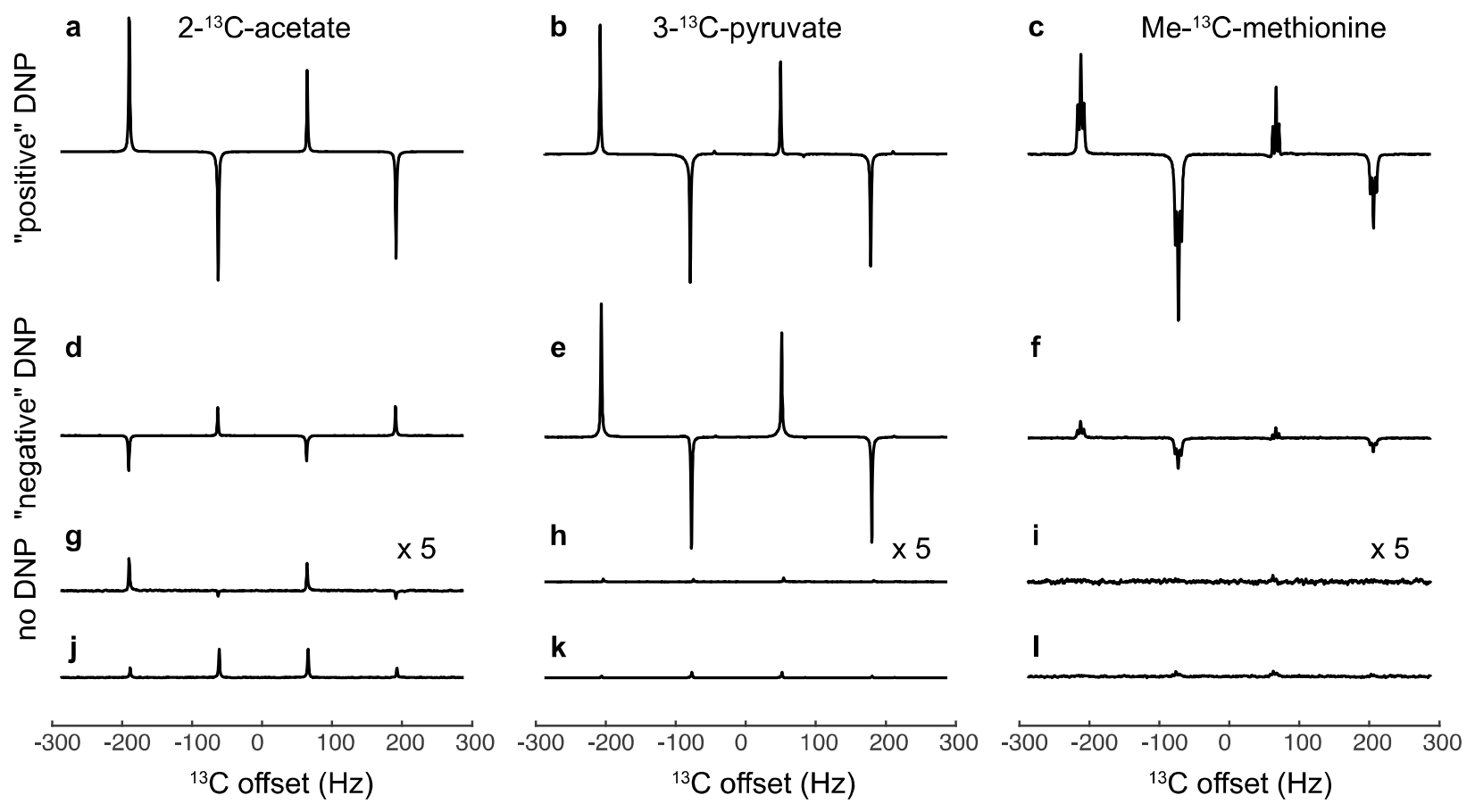

Fig. $3{ }^{13} \mathrm{C}$ 1D spectra of the methyl signal of $2-{ }^{13} \mathrm{C}$-acetate (left), $3-{ }^{13} \mathrm{C}$-pyruvate (middle) and Me- ${ }^{13} \mathrm{C}$-methionine (right) at $11.7 \mathrm{~T}$ and $298 \mathrm{~K}$. Spectra a to $\mathbf{i}$ are obtained using the scheme 
shown in Fig. 2b. For Me- ${ }^{13} \mathrm{C}$-methionine, the first spectrum is shown. For $2-{ }^{13} \mathrm{C}$-acetate, the 10th spectrum is shown; for $3-{ }^{13} \mathrm{C}$-pyruvate, the 4 th spectrum is shown; earlier spectra are contaminated by residual magnetization. The polarization step in the solid state used either positive DNP (a-c), negative DNP (d-f), or no DNP (g-i). Thermal equilibrium spectra acquired with 4 scans are shown in $\mathbf{j}-\mathbf{l}$. The vertical scale is independent for each molecule but is consistent for all the spectra of a given molecule.

A full explanation of these observations is beyond the scope of this preliminary report. Nevertheless, the salient features may be rationalised by considering Boltzmann population distributions within the energy level diagrams of Fig 1, assuming for simplicity that DNP establishes a common temperature across the entire manifold of nuclear Zeeman and tunnelling quantum levels. For brevity, we refer to this common temperature as "spin temperature", bearing in mind that the associated quantum system involves tunnelling energy as well as Zeeman energy. This assumption is plausible in the current case, since prior observations have shown that the thermal contact between the Zeeman system and the lattice, and the tunnelling system and the lattice, are both very weak at cryogenic temperatures, relative to the Zeemantunnelling contact. ${ }^{21}$ Nevertheless, the interpretation given here is merely qualitative, and encounters obvious problems in some cases, for example when negative temperatures are invoked. Although a negative temperature is a valid concept for finite spin systems, which have a finite set of quantum levels, the concept of negative temperature is inherently flawed for tunnelling levels, which are unbounded at high energy. ${ }^{30}$ A fuller understanding will require a more detailed analysis of the quantum dynamics, supported by further experiments, as is the case for conventional DNP processes. ${ }^{31-37}$ There is extensive prior literature on the interaction of electron spins and methyl tunnelling splittings. ${ }^{38-41}$ 
First consider the case where the tunnelling splitting is smaller than the Zeeman splitting (Fig. 1a-b). In this case, the lowest and highest energy level both belong to the A symmetry species. Hence, either a very low positive spin temperature (in which only the lowest energy level is significantly populated, Fig. 1a) or a very low negative spin temperature (so that only the highest energy level is significantly populated, Fig. 1b) both give rise to excess population in the A manifold, compared to the E manifold. We therefore expect that in such systems the sign of the LLS after dissolution, and hence that of the antiphase ${ }^{13} \mathrm{C}$ signals, is independent of the sign of DNP. As shown in Fig. 3c and 3f, this is observed for ${ }^{13} \mathrm{CH}_{3}$-methionine and in $3 \mathrm{~b}$ and $3 \mathrm{e}$ for 3${ }^{13} \mathrm{C}$-pyruvate.

Different behaviour is anticipated when the tunnelling splitting is large compared to the Zeeman splitting (Fig. 1c-d). In this case, the lowest energy level belongs to the A manifold, while the highest energy level belongs to the E manifold. Hence, very low positive or negative spin temperatures are expected to give rise to $\mathrm{A} / \mathrm{E}$ population imbalances of opposite sign. We therefore expect that in such systems, a change in sign of DNP changes the sign of the LLS after dissolution, and hence that of the antiphase ${ }^{13} \mathrm{C}$ signals. As shown in Fig. $3 \mathrm{a}$ and $3 \mathrm{~d}$ this is observed for $2-{ }^{13} \mathrm{C}$-acetate. The reduced intensity of the observed signals may be associated with a breakdown of the spin temperature hypothesis for negative DNP involving unbounded tunnelling levels, as discussed above. The existence of a relatively large tunnelling splitting for $2-{ }^{13} \mathrm{C}$-acetate is consistent with the observation of a small QRIP effect in the absence of microwave irradiation (Fig. 3g).

Table 1 Relaxation time constants (in seconds) for the longitudinal magnetisation and the A/E imbalance at $11.7 \mathrm{~T}$ and $298 \mathrm{~K}$. The longitudinal relaxation rates are obtained as a singleexponential fit to the total area of the multiplet in inversion recovery experiments. The minimum 
and maximum relaxation time constants of the $\mathrm{A} / \mathrm{E}$ imbalance are given, corresponding to the extrema of single-exponential fits of each component in the multiplet in the dissolution NMR experiments shown in Fig. $2 b$.

\begin{tabular}{cccc} 
substance & $T_{1 \mathrm{C}} / \mathrm{s}$ & $T_{1 \mathrm{H}} / \mathrm{s}$ & $T_{\mathrm{AE}} / \mathrm{s}(\min , \max )$ \\
\hline $2-{ }^{13} \mathrm{C}$-acetate & 13.5 & 5.5 & $(46,52)$ \\
$3-{ }^{13} \mathrm{C}$-pyruvate & 13.5 & 5.1 & $(16,19)$ \\
${ }^{13} \mathrm{CH}_{3}-$ methionine & 6.8 & 2.2 & $(5,8)$ \\
$3-{ }^{13} \mathrm{C}$-alanine & 2.1 & 1.4 & $\mathrm{NA}$
\end{tabular}

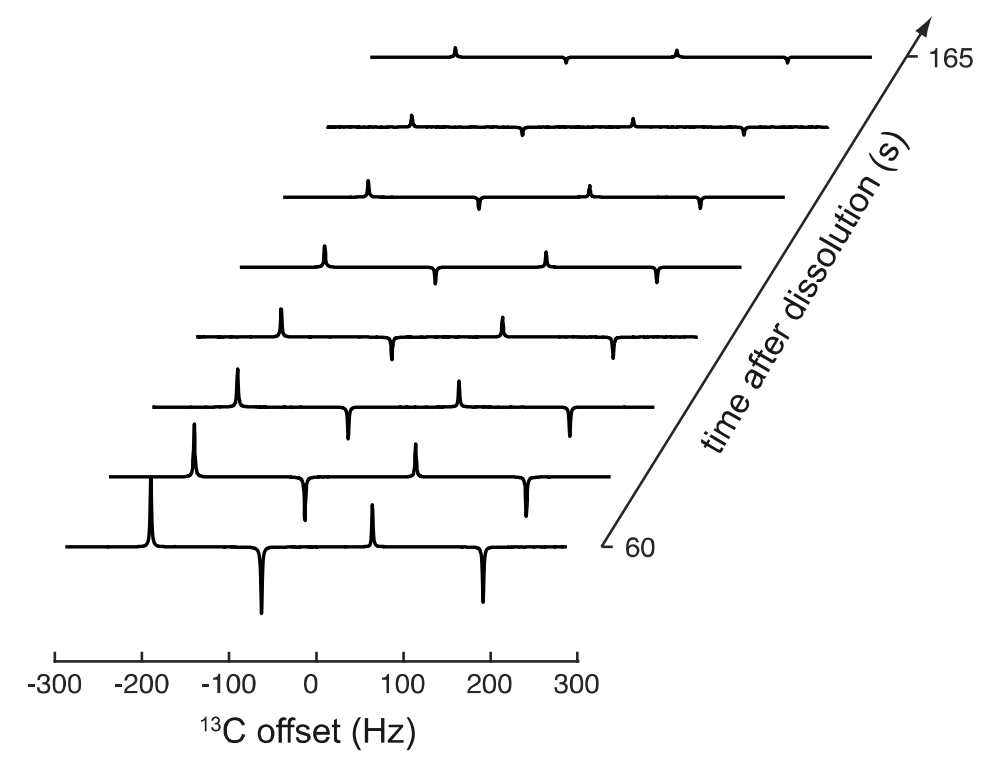

Fig. 4 Selection of ${ }^{13} \mathrm{C}$ 1D spectra of the methyl signal of $2-{ }^{13} \mathrm{C}$-acetate from the time series obtained with the experimental procedure shown in Fig. 2a-b, with positive DNP at $11.7 \mathrm{~T}$ and $298 \mathrm{~K}$. The first spectrum shown here was obtained $\sim 60 \mathrm{~s}$ after dissolution. Subsequent spectra are obtained every $15 \mathrm{~s}$.

The relaxation rate constant of the LLS after dissolution to the liquid state may be obtained from $1 \mathrm{D}{ }^{13} \mathrm{C}$ spectra obtained with the pulse sequence shown in Fig. 2b. Experimental spectra of 
$2-{ }^{13} \mathrm{C}$-acetate are shown in Fig. 4a. Note the long persistence of these enhanced signals, with relaxation times in the range of $T_{L L S}=50 \mathrm{~s}$, relative to the Zeeman relaxation times for $2-{ }^{13} \mathrm{C}$ acetate, which are $T_{1}\left({ }^{13} \mathrm{C}\right)=13.5 \mathrm{~s}$ and $T_{1}\left({ }^{1} \mathrm{H}\right)=5.5 \mathrm{~s}$. Table 1 summarises the relaxation time constants of the LLS, together with longitudinal relaxation time constants for ${ }^{1} \mathrm{H}$ and ${ }^{13} \mathrm{C}$ magnetization. In all cases where antiphase multiplets were detected, $T_{\mathrm{LLS}}$ is found to be longer than $T_{1}\left({ }^{1} \mathrm{H}\right)$. For $3-{ }^{13} \mathrm{C}$-pyruvate and ${ }^{13} \mathrm{CH}_{3}$-methionine, however, the relaxation time $T_{\mathrm{LLS}}$ of the $\mathrm{A} / \mathrm{E}$ imbalance is found to be comparable to $T_{1}\left({ }^{13} \mathrm{C}\right)$. The AEI is nevertheless long lived, since it involves ${ }^{1} \mathrm{H}$ spin order that relaxes more slowly than ${ }^{1} \mathrm{H}$ magnetization, but it does not extend the timescales that may be probed with ${ }^{13} \mathrm{C}$ magnetization. On the other hand, for acetate $T_{\mathrm{LLS}}$ is found to be larger than both $T_{1}\left({ }^{1} \mathrm{H}\right)$ and $T_{1}\left({ }^{13} \mathrm{C}\right)$. The long lifetime of the LLS for $2-{ }^{13} \mathrm{C}$-acetate in solution may be explained by the low rotational barrier for the methyl group in this compound, which gives rise to a short correlation time for methyl rotation in solution ${ }^{17-18}$. At this point, it is not known whether the radical content of the dissolved solution significantly influences the decay of the methyl long-lived states. Paramagnetic effects on the relaxation of 2-spin long-lived states are generally weaker than for conventional magnetization. ${ }^{42}$

The absence of any hyperpolarized antiphase ${ }^{13} \mathrm{C}$ multiplet in our dissolution-NMR experiments on $3-{ }^{13} \mathrm{C}$-alanine may be due to the fact that the methyl group is bound to an $\mathrm{sp}^{3}$ carbon, a configuration which is known to result in a large rotational barrier. ${ }^{43}$ Strong hindering of methyl rotation may destroy the long-lived polarization effects, for at least two main reasons. Firstly, the symmetry-adapted basis states, with their A and E symmetry labels, may not be accurate energy eigenstates for frozen methyl groups in the solid state. It is therefore debatable as to whether the AEI is established in the solid state in this case; Secondly, as shown by theory ${ }^{17-}$ ${ }^{18}$, a large value for the rotational correlation time $\tau_{\mathrm{R}}$ of the methyl group is associated with a 
short AEI decay time constant in solution. Hence, in $3-{ }^{13} \mathrm{C}$-alanine, the methyl AEI, even if it is generated by DNP in the solid state, may be too short-lived to survive the dissolution, transfer and injection process. Further experiments are needed to resolve these issues.

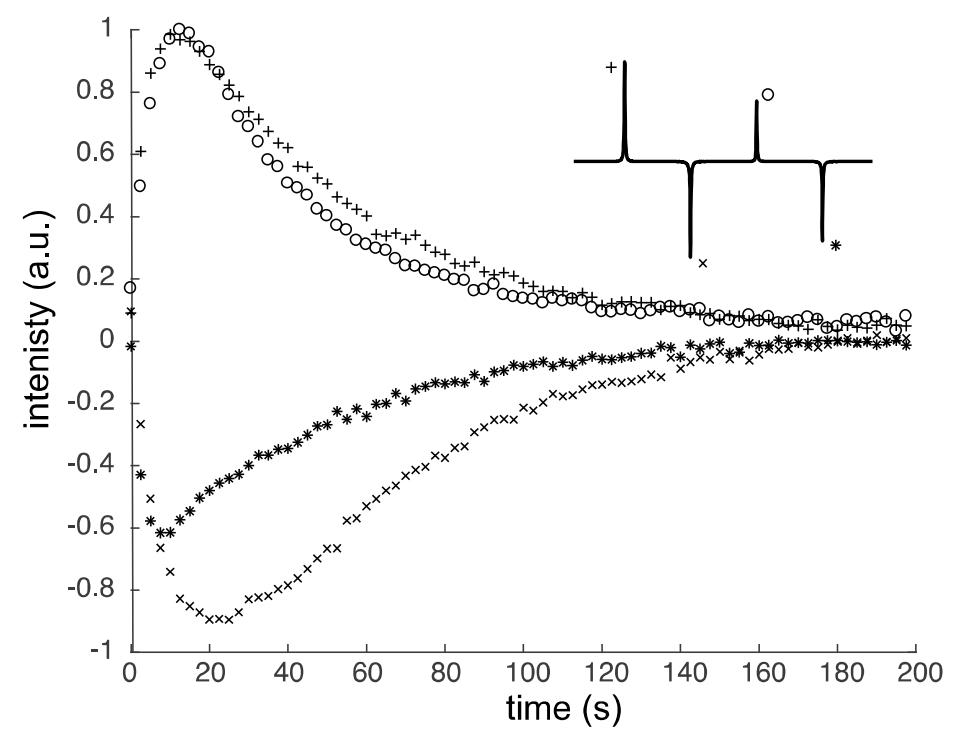

Fig. 5 Intensities of the components of the hyperpolarized antiphase signal in a dissolution-NMR experiment on $2-{ }^{13} \mathrm{C}$-acetate at $11.7 \mathrm{~T}$ and $298 \mathrm{~K}$, with positive DNP. The acquisition was performed using the pulse sequence in Fig. $2 \mathrm{c}$. The time axis starts with the last $T_{00}$ filter.

Figure 5 shows the build-up and decay of the four antiphase components of the ${ }^{13} \mathrm{C}$ multiplet of $2-{ }^{13} \mathrm{C}$-acetate, monitored with a series of experiments with small-tip-angle pulses, using a train of $T_{00}$ filters after dissolution (see Fig. 2c). The build-up occurs on a time scale that is comparable with $T_{1}\left({ }^{1} \mathrm{H}\right)$. These trajectories are similar to those obtained in the QRIP observations on $\gamma$-picoline, which have been thoroughly analysed in terms of ${ }^{1} \mathrm{H}-{ }^{13} \mathrm{C}$ dipolar and CSA cross-relaxation effects for the case of a rapidly rotating methyl group. ${ }^{17}$

Table 2 DNP-induced enhancements in dissolution NMR experiments of methyl- ${ }^{13} \mathrm{C}$-molecules. The enhancements are expressed as ratios of the area of $n^{\text {th }}$ component in the hyperpolarized quartet to that of the corresponding component in the thermal equilibrium spectrum. The 
components of the multiplets are numbered from left to right. The enhancement for 1 scan is given for the spectrum shown in Fig. 3a-c. The summed enhancements are given for 20, 4 and 10 consecutive scans for $2-{ }^{13} \mathrm{C}$-acetate, Me- ${ }^{13} \mathrm{C}$-methionine and $3-{ }^{13} \mathrm{C}$-pyruvate, respectively

\begin{tabular}{ccccccccc} 
& \multicolumn{3}{c}{ enhancements (for 1 scan) } & \multicolumn{5}{c}{ summed enhancements } \\
substance & 1 & 2 & 3 & 4 & 1 & 2 & 3 & 4 \\
\hline $2-{ }^{13} \mathrm{C}$-acetate & 62 & -20 & 11 & -47 & 570 & -154 & 115 & -410 \\
$3-{ }^{13} \mathrm{C}$-pyruvate & 274 & -110 & 48 & -244 & 1020 & -380 & 191 & -867 \\
${ }^{13} \mathrm{CH}_{3}$-methionine & 172 & -119 & 21 & -150 & 281 & -179 & 44 & -233
\end{tabular}

The enhancements of the anti-phase signals shown in Fig. 3a-c are reported in Table 2. The enhancements are significantly smaller than those achieved by direct polarization in dissolutionDNP experiments. This is mainly because observable population differences across the ${ }^{13} \mathrm{C}$ transitions are only progressively released from the LLS by cross-relaxation processes. This may be seen either as a limitation (smaller enhancements) or as an advantage (repeated observations) of methyl LLS as carriers of hyperpolarized spin order. The enhanced Zeeman magnetization generated in conventional dissolution DNP experiments is completely destroyed when a single $90^{\circ}$ pulse is applied to generate observable NMR signals. This is not the case for the experiments described here, since the observation pulses do not influence the methyl LLS, allowing repeated observations by "harvesting" the LLS in small fractions. Table 2 also shows figures for the enhancements obtained when the entire time series of signals is integrated. This cumulative enhancement provides qualitative information on the degree of hyperpolarization stored in and released by the $\mathrm{A} / \mathrm{E}$ imbalance (note that if $\mathrm{N}$ consecutive spectra in the time series were summed, the increase in signal to noise ratio (SNR) would be given by the cumulative 
enhancement divided by $\sqrt{N}$. Since the antiphase magnetisation builds up on a time-scale comparable to $\mathrm{T}_{1}$, the SNR could be optimised with Ernst-Angle-type excitation ${ }^{44}$ ).

In summary, we have demonstrated that long-lived nuclear spin state imbalances in methyl groups may be generated by dynamic nuclear polarization. The signs of the resulting hyperpolarized antiphase ${ }^{13} \mathrm{C}$ signals are explained by invoking the spin-temperature hypothesis, taking into account the distribution of energy levels. The relaxation time constants of methyl LLSs are found to be strongly dependent on the molecular environment, but are often longer than the ${ }^{1} \mathrm{H}$ or ${ }^{13} \mathrm{C}$ spin-lattice relaxation times constants. Methyl long-lived states may provide a new class of relaxation-based probes to characterize molecular dynamics, and serve as carriers of hyperpolarized spin order, facilitating applications to NMR investigations of biochemistry and metabolism, and in molecular imaging.

\section{AUTHOR INFORMATION}

\section{Notes}

The authors declare no competing financial interests.

\section{ACKNOWLEDGMENT}

The authors thank Lyndon Emsley for making means available. The research leading to these results has received funding from COST (CA15209), Royal Society CNRS (grant code IE140525), EPSRC-UK (grant codes EP/N002482/1, EP/P009980/1), the European Research Council under the European Union's Horizon 2020 research and innovation programme (ERC Grant Agreement n 714519 / HP4all), the ERC grant "Dilute para-water", the EPFL, the Swiss National Science Foundation, and Bruker BioSpin SA Switzerland.

\section{REFERENCES}


1. Ardenkjaer-Larsen, J. H.; Fridlund, B.; Gram, A.; Hansson, G.; Hansson, L.; Lerche, M. H.; Servin, R.; Thaning, M.; Golman, K., Increase in Signal-to-Noise Ratio of $>10,000$ Times in Liquid-State Nmr. Proc. Natl. Acad. Sci. U.S.A. 2003, 100 (18).

2. Adams, R. W.; Aguilar, J. A.; Atkinson, K. D.; Cowley, M. J.; Elliott, P. I. P.; Duckett, S. B.; Green, G. G. R.; Khazal, I. G.; Lopez-Serrano, J.; Williamson, D. C., Reversible Interactions with Para-Hydrogen Enhance Nmr Sensitivity by Polarization Transfer. Science 2009, 323 (5922), 1708.

3. Bowers, C. R.; Weitekamp, D. P., Para-Hydrogen and Synthesis Allow Dramatically Enhanced Nuclear Alignment L.Am. Chem.Soc. 1987, 109 (18), 5541-5542.

4. Carravetta, M.; Johannessen, O. G.; Levitt, M. H., Beyond the T-1 Limit: Singlet Nuclear Spin States in Low Magnetic Fields. Phvs. Rev._Lett. 2004, 92 (15), 153003.

5. Carravetta, M.; Levitt, M. H., Long-Lived Nuclear Spin States in High-Field Solution Nmr. L.Am.Chem.Soc. 2004, 126 (20), 6228-6229.

6. Levitt, M. H., Singlet Nuclear Magnetic Resonance. Annu. Rev. Phys. Chem. 2012, 63, 89-105.

7. Pileio, G.; Bowen, S.; Laustsen, C.; Tayler, M. C. D.; Hill-Cousins, J. T.; Brown, L. J.; Brown, R. C. D.; Ardenkjaer-Larsen, J. H.; Levitt, M. H., Recycling and Imaging of Nuclear Singlet Hyperpolarization. J.Am. Chem.Soc. 2013, 135 (13), 5084-5088.

8. $\quad$ Bornet, A.; Ji, X.; Mammoli, D.; Vuichoud, B.; Milani, J.; Bodenhausen, G.; Jannin, S., Long-Lived States of Magnetically Equivalent Spins Populated by Dissolution-Dnp and Revealed by Enzymatic Reactions. Chem.Eur.J. 2014, 20 (51), 17113-17118.

9. Buratto, R.; Bornet, A.; Milani, J.; Mammoli, D.; Vuichoud, B.; Salvi, N.; Singh, M.; Laguerre, A.; Passemard, S.; Gerber-Lemaire, S.; Jannin, S.; Bodenhausen, G., Drug Screening Boosted by Hyperpolarized Long-Lived States in Nmr. ChemMedChem 2014, 9 (11), 2509-2515. 10. Vasos, P. R.; Comment, A.; Sarkar, R.; Ahuja, P.; Jannin, S.; Ansermet, J. P.; Konter, J. A.; Hautle, P.; van den Brandt, B.; Bodenhausen, G., Long-Lived States to Sustain Hyperpolarized Magnetization. Proc.Natl.Acad Sci.U.S.A. 2009, 106 (44), 18469-18473. 11. Warren, W. S.; Jenista, E.; Branca, R. T.; Chen, X., Increasing Hyperpolarized Spin Lifetimes through True Singlet Eigenstates. Science (New York. N.Y.) 2009, 323, 1711-4.

12. Franzoni, M. B.; Buljubasich, L.; Spiess, H. W.; M,nnemann, K., Long-Lived 1h Singlet Spin States Originating from Para-Hydrogen in Cs-Symmetric Molecules Stored for Minutes in High Magnetic Fields. J. Am. Chem. Soc. 2012, 134, 10393-6.

13. Roy, S. S.; Rayner, P. J.; Norcott, P.; Green, G. G. R.; Duckett, S. B., Long-Lived States to Sustain Sabre Hyperpolarised Magnetisation. Phvs. Chem. Chem. Phvs. 2016, 18 (36), 2490524911.

14. Theis, T.; Ortiz, G. X.; Logan, A. W. J.; Claytor, K. E.; Feng, Y.; Huhn, W. P.; Blum, V.; Malcolmson, S. J.; Chekmenev, E. Y.; Wang, Q.; Warren, W. S., Direct and Cost-Efficient Hyperpolarization of Long-Lived Nuclear Spin States on Universal N-15(2)-Diazirine Molecular Tags. Science Advances 2016, 2 (3).

15. Eills, J.; Stevanato, G.; Bengs, C.; Gloggler, S.; Elliott, S. J.; Alonso-Valdesueiro, J.; Pileio, G.; Levitt, M. H., Singlet Order Conversion and Parahydrogen-Induced Hyperpolarization of C-13 Nuclei in near-Equivalent Spin Systems. L.Magn. Reson. 2017, 274, 163-172.

16. Stevanato, G.; Hill-Cousins, J. T.; Hakansson, P.; Roy, S. S.; Brown, L. J.; Brown, R. C. D.; Pileio, G.; Levitt, M. H., A Nuclear Singlet Lifetime of More Than One Hour in RoomTemperature Solution. Angew. Chem.Int.Ed. 2015, 54 (12), 3740-3743. 
17. Dumez, J.-N.; Hakansson, P.; Mamone, S.; Meier, B.; Stevanato, G.; Hill-Cousins, J. T.; Roy, S. S.; Brown, R. C. D.; Pileio, G.; Levitt, M. H., Theory of Long-Lived Nuclear Spin States in Methyl Groups and Quantum-Rotor Induced Polarisation. J. Chem. Phys. 2015, 142 (4), 044506.

18. Meier, B.; Dumez, J.-N.; Stevanato, G.; Hill-Cousins, J. T.; Roy, S. S.; Hakansson, P.; Mamone, S.; Brown, R. C. D.; Pileio, G.; Levitt, M. H., Long-Lived Nuclear Spin States in Methyl Groups and Quantum-Rotor-Induced Polarization. J. Am. Chem. Soc. 2013, 135 (50), 18746-18749.

19. Icker, M.; Berger, S., Unexpected Multiplet Patterns Induced by the Haupt-Effect. $J$. Magn. Reson. 2012, 219, 1-3.

20. Icker, M.; Fricke, P.; Grell, T.; Hollenbach, J.; Auer, H.; Berger, S., Experimental Boundaries of the Quantum Rotor Induced Polarization (Qrip) in Liquid State Nmr. Magn. Reson. Chem. 2013, 51 (12), 815-820.

21. Horsewill, A. J., Quantum Tunnelling Aspects of Methyl Group Rotation Studied by Nmr. Prog. Nucl. Magn. Reson. Spectrosc. 1999, 35 (4), 359-389.

22. Wenckebach, T., Essentials of Dynamic Nuclear Polarization. Spindrift Publications: 2016.

23. Tayler, M. C. D.; Marco-Rius, I.; Kettunen, M. I.; Brindle, K. M.; Levitt, M. H.; Pileio, G., Direct Enhancement of Nuclear Singlet Order by Dynamic Nuclear Polarization. J. Am. Chem. Soc. 2012, 134, 7668-71.

24. Mammoli, D.; Vuichoud, B.; Bornet, A.; Milani, J.; Dumez, J.-N.; Jannin, S.; Bodenhausen, G., Hyperpolarized Para-Ethanol. J. Phys. Chem. B 2015.

25. Jhajharia, A.; Weber, E. M. M.; Kempf, J. G.; Abergel, D.; Bodenhausen, G.; Kurzbach, D., Communication: Dissolution Dnp Reveals a Long-Lived Deuterium Spin State Imbalance in Methyl Groups. J. Chem. Phys. 2017, 146 (4).

26. Jannin, S.; Bornet, A.; Melzi, R.; Bodenhausen, G., High Field Dynamic Nuclear Polarization at 6.7 T: Carbon-13 Polarization above 70\% within 20 Min. Chem. Phys. Lett. 2012, 549, 99-102.

27. Bornet, A.; Milani, J.; Vuichoud, B.; Linde, A. J. P.; Bodenhausen, G.; Jannin, S., Microwave Frequency Modulation to Enhance Dissolution Dynamic Nuclear Polarization. Chem. Phys. Lett. 2014, 602, 63-67.

28. Bornet, A.; Jannin, S., Optimizing Dissolution Dynamic Nuclear Polarization. J. Magn. Reson. 2016, 264, 13-21.

29. Tayler, M. C. D.; Levitt, M. H., Accessing Long-Lived Nuclear Spin Order by IsotopeInduced Symmetry Breaking. J. Am. Chem. Soc. 2013, 135, 2120-3.

30. Abragam, A.; Goldman, M., Nuclear Magnetism: Order and Disorder. Oxford, 1982.

31. Hovav, Y.; Feintuch, A.; Vega, S., Theoretical Aspects of Dynamic Nuclear Polarization in the Solid State - the Solid Effect. J. Magn. Reson. 2010, 207 (2), 176-189.

32. Hovav, Y.; Feintuch, A.; Vega, S., Theoretical Aspects of Dynamic Nuclear Polarization in the Solid State - the Cross Effect. J. Magn. Reson. 2012, 214, 29-41.

33. Hovav, Y.; Feintuch, A.; Vega, S., Theoretical Aspects of Dynamic Nuclear Polarization in the Solid State - Spin Temperature and Thermal Mixing. Phys. Chem. Chem. Phys. 2013, 15 (1), 188-203.

34. Hovav, Y.; Levinkron, O.; Feintuch, A.; Vega, S., Theoretical Aspects of Dynamic Nuclear Polarization in the Solid State: The Influence of High Radical Concentrations on the Solid Effect and Cross Effect Mechanisms. Appl. Magn. Reson. 2012, 43 (1-2), 21-41. 
35. Shimon, D.; Hovav, Y.; Feintuch, A.; Goldfarb, D.; Vega, S., Dynamic Nuclear Polarization in the Solid State: A Transition between the Cross Effect and the Solid Effect. Phys. Chem. Chem. Phys. 2012, 14 (16), 5729-5743.

36. Caracciolo, F.; Filibian, M.; Carretta, P.; Rosso, A.; De Luca, A., Evidence of SpinTemperature in Dynamic Nuclear Polarization: An Exact Computation of the Epr Spectrum. Phys. Chem. Chem. Phys. 2016, 18 (36), 25655-25662.

37. Serra, S. C.; Filibian, M.; Carretta, P.; Rosso, A.; Tedoldi, F., Relevance of Electron Spin Dissipative Processes to Dynamic Nuclear Polarization Via Thermal Mixing. Phys. Chem. Chem. Phys. 2014, 16 (2), 753-764.

38. Clough, S.; Horsewill, A. J.; Paley, M. N. J., Dynamic Nuclear Polarization by the Cooling of Tunnelling Methyl Groups. Journal of Physics C-Solid State Physics 1982, 15 (17), 3803-3808.

39. Clough, S.; Mulady, B. J., Tunnelling Resonances in Proton Spin-Lattice Relaxation Phys. Rev. Lett. 1973, 30 (5), 161-163.

40. Freed, J. H., Quantum Effects of Methyl-Group Rotations in Magnetic Resonance - Esr Splittings and Linewidths. J. Chem. Phys. 1965, 43 (5), 1710-\&.

41. Horsewill, A. J.; Abu-Khumra, S. M. M., Dynamic Tunneling Polarization as a Quantum Rotor Analogue of Dynamic Nuclear Polarization and the Nmr Solid Effect. Phys. Rev. Lett. 2011, 107 (12).

42. Tayler, M. C. D.; Levitt, M. H., Paramagnetic Relaxation of Nuclear Singlet States. Physical chemistry chemical physics : PCCP 2011, 13, 9128-30.

43. Prager, M.; Heidemann, A., Rotational Tunneling and Neutron Spectroscopy: A Compilation. Chem. Rev. 1997, 97 (8), 2933-2966.

44. $\quad$ Ernst, R. R.; Bodenhausen, G.; Wokaun, A., Principles of Nuclear Magnetic Resonance in One and Two Dimensions. Oxford University Press: Oxford, 1990. 
a

b

个 Energy

C
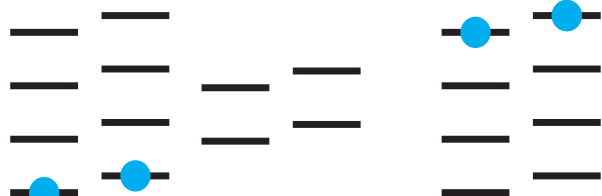

d
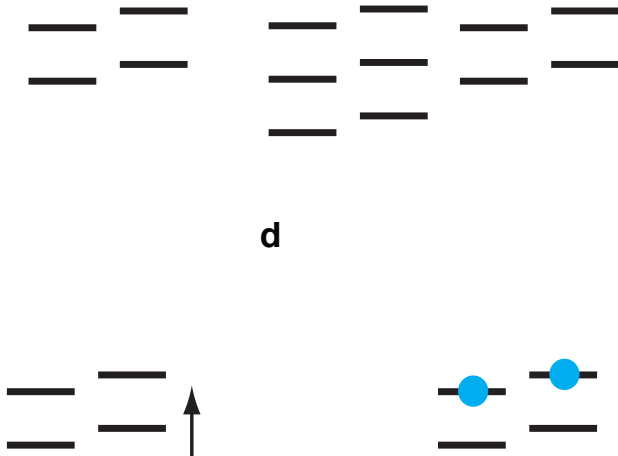

$\Delta E_{\text {tunnel }}$
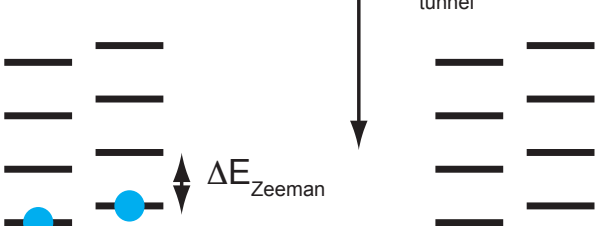
A
$E_{a}, E_{b}$

A

$E_{a}, E_{b}$ 


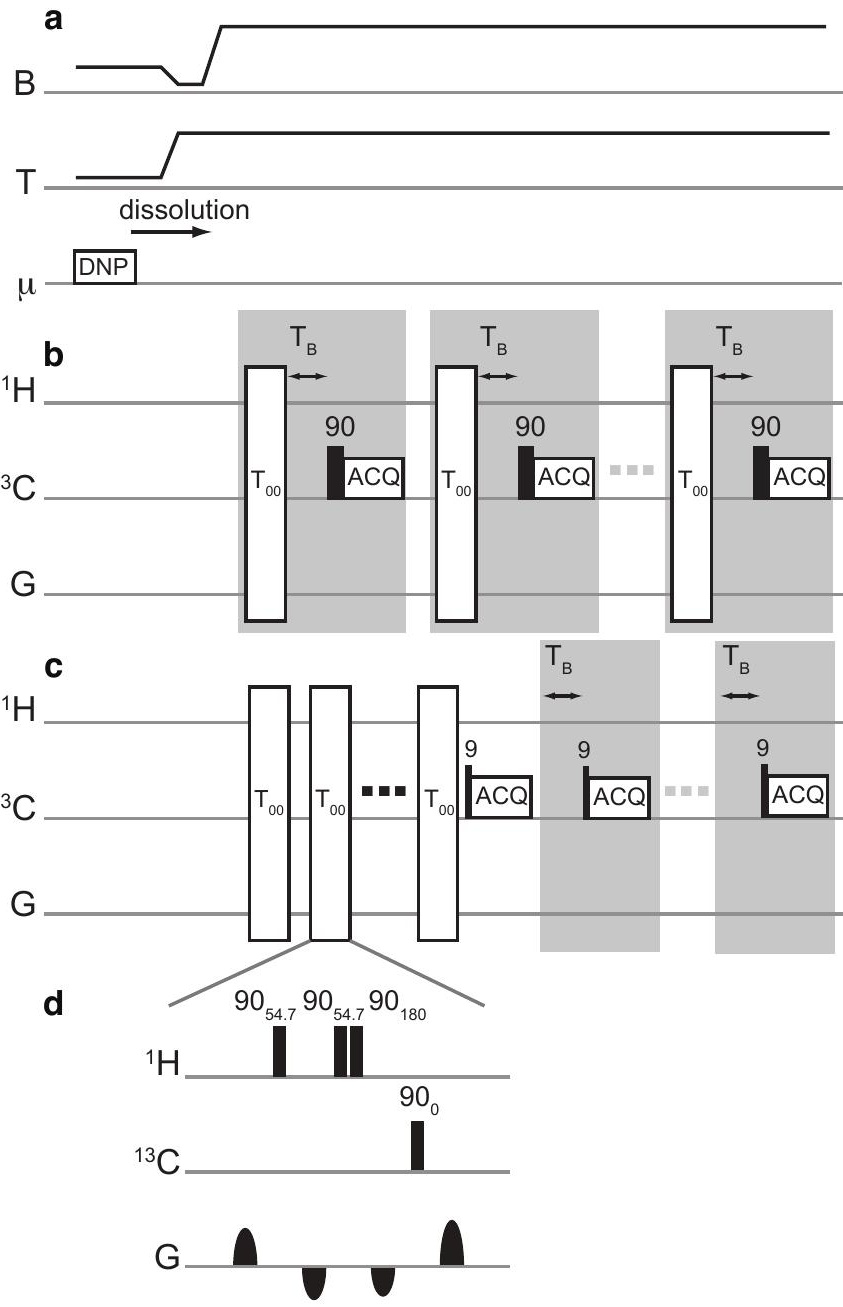




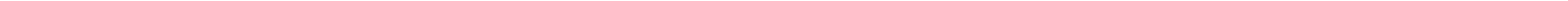




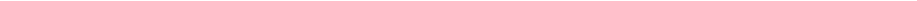




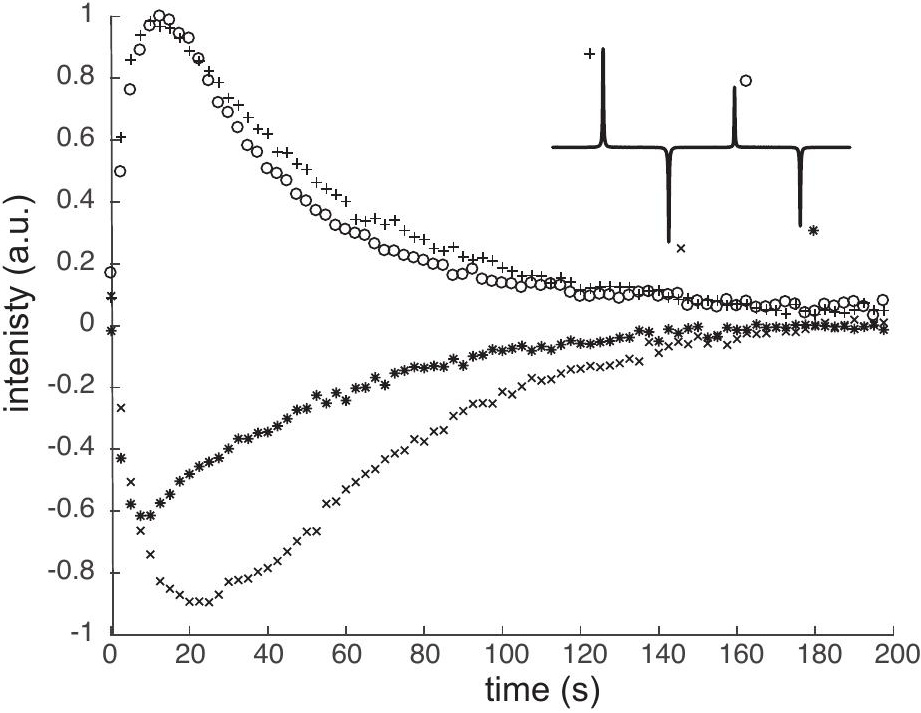




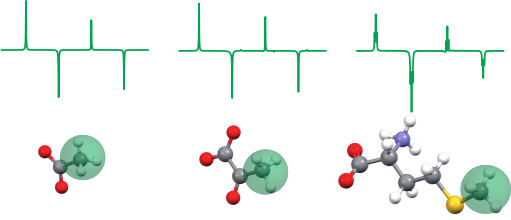

